\title{
Space-like maximal surfaces containing entire null lines in Lorentz-Minkowski 3-space
}

\author{
By Shintaro Akamine, ${ }^{*)}$ Masaaki UmeharA**) and Kotaro YamadA ${ }^{* * *}$ ) \\ (Communicated by Kenji FukaYA, M.J.A., Oct. 15, 2019)
}

\begin{abstract}
Consider a surface $S$ immersed in the Lorentz-Minkowski 3 -space $\boldsymbol{R}_{1}^{3}$. A complete light-like line in $\boldsymbol{R}_{1}^{3}$ is called an entire null line on the surface $S$ in $\boldsymbol{R}_{1}^{3}$ if it lies on $S$ and consists of only null points with respect to the induced metric. In this paper, we show the existence of embedded space-like maximal graphs containing entire null lines. If such a graph is defined on a convex domain in $\boldsymbol{R}^{2}$, then it must be contained in a light-like plane (cf. Remark 3.3). Our example is critical in the sense that it is defined on a certain non-convex domain.
\end{abstract}

Key words: Maximal surface; type change; zero mean curvature; Lorentz-Minkowski space.

1. Introduction. We let $\boldsymbol{R}_{1}^{3}$ be the LorentzMinkowski 3-space of signature $(++-)$. It is wellknown that there are no complete space-like zero mean curvature immersions in $\boldsymbol{R}_{1}^{3}$ other than planes (cf. [5]). It is thus interesting to investigate singularities of zero mean curvature surfaces. In fact, maximal surfaces with singularities were investigated by several geometers, see references in [11]. Regarding these works, the second and third authors introduced the concept of maxface in [11], which covers a large class of space-like surfaces with zero mean curvature admitting singular points. The elliptic catenoid

$$
f_{0}:=(\sinh v \cos u, \sinh v \sin u, v) \quad(|u| \leq \pi, v \in \boldsymbol{R})
$$

is a typical example of maxface with a cone-like singular point (cf. Fig. 1, left).

However, if space-like maximal surfaces have analytic extensions which change their causal type, then those extensions cannot remain in the class of maxfaces. In [13], two functions $A_{f}$ and $B_{f}$ for immersed hypersurfaces in Lorentzian manifolds were introduced. Using them, we give here a new notion 'ZMC-map' as an appropriate new class for

2010 Mathematics Subject Classification. Primary 53A10, 53B30; Secondary 35M10.

*) Graduate School of Mathematics, Nagoya University, Furocho Chikusa-ku, Nagoya 464-8602, Japan.

**) Department of Mathematical and Computing Sciences, Tokyo Institute of Technology, 2-12-1-W8-34, Ookayama Meguro-ku, Tokyo 152-8552, Japan.

***) Department of Mathematics, Tokyo Institute of Technology, 2-21-1-H-7, Ookayama Meguro-ku, Tokyo 152-8551, Japan. zero mean curvature surfaces with singularities, as follows (maxfaces are all ZMC-maps but the converse is not true): Let $U$ be a domain in the $u v$-plane, and let $f: U \rightarrow \boldsymbol{R}_{1}^{3}$ be a smooth map into the Lorentz-Minkowski 3-space $\boldsymbol{R}_{1}^{3}$. We set

$$
P:=\left(\begin{array}{ll}
f_{u} \cdot f_{u} & f_{u} \cdot f_{v} \\
f_{v} \cdot f_{u} & f_{v} \cdot f_{v}
\end{array}\right)
$$

and

$$
B_{f}:=\operatorname{det}(P),
$$

where - denotes the canonical Lorentzian product of $\boldsymbol{R}_{1}^{3}$ and $\operatorname{det}(P)$ denotes the determinant of the $2 \times 2$ matrix $P$. We set

$$
Q:=\left(\begin{array}{cc}
f_{u u} \cdot \tilde{\nu} & f_{u v} \cdot \tilde{\nu} \\
f_{v u} \cdot \tilde{\nu} & f_{v v} \cdot \tilde{\nu}
\end{array}\right),
$$

where

$$
\tilde{\nu}:=\left(\begin{array}{ccc}
1 & 0 & 0 \\
0 & 1 & 0 \\
0 & 0 & -1
\end{array}\right)\left(f_{u} \times_{E} f_{v}\right)
$$

and $f_{u} \times_{E} f_{v}$ denotes the Euclidean vector product of $\boldsymbol{R}^{3}$. Here $\tilde{\nu}$ gives a Lorentzian normal vector field of $f$ defined on $U$. Consider the matrix

$$
W:=\tilde{P} Q
$$

and set

$$
A_{f}:=\operatorname{trace}(W),
$$

where $\tilde{P}$ is the cofactor matrix of $P$. We call $f$ a $Z M C$-map (i.e. zero mean curvature map) if it is an immersion on an open dense subset of $U$ and $A_{f}$ 

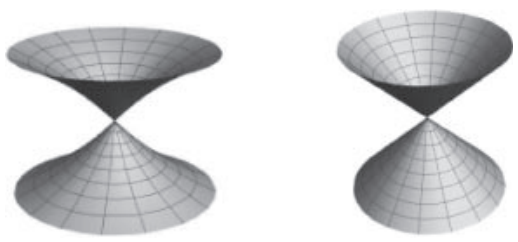

Fig. 1. The elliptic catenoid (left) and the light-cone (right).

vanishes identically. Since the property that $A_{f}$ varnishes is independent of the choice of local coordinates, ZMC-maps can be defined from an arbitrarily given 2-manifold. Moreover, one can generalize the concept of ZMC-map for a map into $\boldsymbol{R}_{1}^{n+1}$ from an arbitrarily given $n$-manifold using the function $A_{f}$ given in [13].

We let $f$ be a ZMC-map. A point $p \in U$ is said to be a space-like point (resp. time-like point) of $f$, if $B_{f}(p)>0$ (resp. $\left.B_{f}(p)<0\right)$. A point which is neither space-like nor time-like is said to be a null point or a light-like point of $f$. (If $p$ is a singular point of $f$, then $B_{f}(p)=0$. So $p$ is a null point.)

If $f: U \rightarrow \boldsymbol{R}_{1}^{3}$ is a light-like surface, that is, all points on $U$ are null points, then $A_{f}$ vanishes identically (cf. [9, Example 4]). So, by our definition, a light-like surface is also an example of a ZMC-map.

Definition 1.1. A ZMC-map is said to be maximal type (resp. of mixed type) if $B_{f} \geq 0$ but does not vanish identically (resp. $B_{f}$ changes its sign on $U$ ). On the other hand, a ZMC-map is said to be null or light-like if $B_{f}$ vanishes identically. A ZMC-map which is neither maximal, of mixed type nor light-like is said to be time-like.

We now fix $f: U \rightarrow \boldsymbol{R}_{1}^{3}$ as a ZMC-map. A null point $p \in U$ is called degenerate if the exterior derivative of $B_{f}$ vanishes at $p$. The light-cone

$$
f_{1}:=(v \cos u, v \sin u, v) \quad(|u| \leq \pi, v \in \boldsymbol{R}),
$$

is a light-like ZMC-map consisting only of degenerate null points.

Definition 1.2. We say that a ZMC-map $f: U \rightarrow \boldsymbol{R}_{1}^{3}$ contains a null line segment if there exists an open interval $I$ and a smooth curve $\gamma: I \rightarrow U$ such that $\gamma(I)$ consists only of null points and $f \circ \gamma(I)$ is a subset of a light-like line in $\boldsymbol{R}_{1}^{3}$. In this case, $f \circ \gamma(I)$ is called a null line. Moreover, if $f \circ \gamma(I)$ coincides with a complete light-like line, we call it an entire null line.
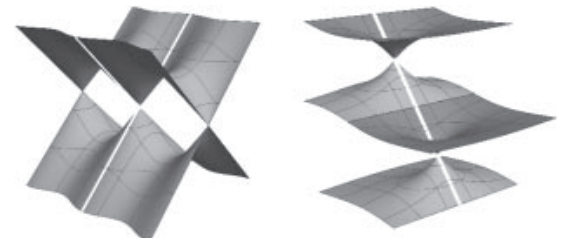

Fig. 2. Maximal surfaces with cone-like singular points lying on null lines, where the white lines indicate null points.

Klyachin [9] showed the following fact:

Fact 1.3 (The line theorem for ZMC-surfaces). Let $f: U \rightarrow \boldsymbol{R}_{1}^{3}$ be a $Z M C$-immersion such that $o \in U$ is a degenerate null point. Then, $f$ contains a null line segment passing through $f(o)$.

This fact was generalized to a much wider class of surfaces, including real analytic constant mean curvature surfaces in $\boldsymbol{R}_{1}^{3}$, see $[12,13]$. Although there are properly embedded time-like ZMC-surfaces with an entire null line (see [3, Examples 2.2 and 2.3]), each of all examples of ZMC-surfaces with space-like points given in $[1,2,6,10]$ containing an entire null line $L$ has at least one cone-like singular point on $L$ (see Fig. 2).

For example, Fig. 2, left is the hyperbolic catenoid (as a ZMC-map) given in [10]. (This surface is called the catenoid of 2nd kind in [10]) and satisfies

$$
\sin ^{2} x+y^{2}=t^{2},
$$

where $(x, y, t)$ is the canonical coordinate system of $\boldsymbol{R}_{1}^{3}$, and $y= \pm t, x=k \pi(k \in \boldsymbol{Z})$ are entire null lines on the surface.

On the other hand, the maximal ZMC-surface given in [1, Theorem 5.3 (1-i)] satisfies

$$
2(y-t) \sin t=\left(x^{2}+y^{2}-2 y t+t^{2}\right) \cos t
$$

and $y=t, x=0$ gives the entire null line on the surface (see Fig. 2, right). By (1) and (2), these two surfaces have no self-intersections.

The purpose of this paper is to show the following

Theorem 1.4. There exist embedded maximal ZMC-surfaces (resp. embedded ZMC-surfaces of mixed type) each of which contains an entire null line.

Remark 1.5. This theorem gives the first example of maximal ZMC-immersion containing an entire null line. On the other hand, examples of ZMC-maps of mixed type containing an entire null 
line were given in [8, Example 9.3 for $m=1$ ] using a different approach.

Corollary 1.6. There exists a family of embedded maximal ZMC-hypersurfaces (resp. embedded ZMC-hypersurfaces of mixed type) in LorentzMinkowski space $\boldsymbol{R}_{1}^{n+1}$ each of which contains an $(n-1)$-dimensional light-like plane.

The proof is completely same as in the proof of [7, Corollary 1.2]. The strategy to prove Theorem 1.4 is as follows: In [7], local existence of a 1parameter family $\left\{f_{c}\right\}$ of ZMC-surfaces of mixed type such that each $f_{c}$ contains a null line segment was shown. By improving the argument there, we will show in Section 3 that each $f_{c}$ can be analytically extended so that it contains an entire null line. Also, by modifying the estimates in [7], we will also show the existence of ZMC-surfaces of maximal type each of which contains an entire null line. Unfortunately, our construction is local, and so the resulting surfaces are not proper. So the following question still remains (this is essentially the same question as in [3, Question 1]):

Question. Are there properly embedded maximal surfaces in $\boldsymbol{R}_{1}^{3}$, which contain at least one entire null line?

As a partial answer of this question, a Bernstein-type theorem for entire ZMC-graphs consisting only of space-like or null points was given in the authors' previous work [3]. As we have mentioned in Remark 1.5, Hashimoto and Kato [8] recently gave a new method for constructing ZMCmaps containing null lines, using bi-complex extensions. The authors expect that this could be developed to apply to the above question.

2. ZMC-surfaces with null lines. We consider a real analytic ZMC-immersion $f$ containing the entire null line in $\boldsymbol{R}_{1}^{3}$. By a suitable Lorentzian transformation of the ambient space, we may assume that this entire null line is given by

$$
L:=\left\{(0, y, y) \in \boldsymbol{R}_{1}^{3} ; y \in \boldsymbol{R}\right\} .
$$

On a neighborhood of a null point, there exist a domain $U$ in the $x y$-plane containing the $y$-axis and a real analytic function $\psi: U \rightarrow \boldsymbol{R}$ such that $f$ can be expressed as the graph of a function of the form

$$
\psi(x, y):=y+\frac{\alpha(y)}{2} x^{2}+\sum_{k=3}^{\infty} \frac{\beta_{k}(y)}{k} x^{k},
$$

where $\alpha$ and $\beta_{k}(k=3,4,5, \ldots)$ are certain real analytic functions on $\boldsymbol{R}$. Since

$$
f(x, y)=(x, y, \psi(x, y)),
$$

we have

$$
\begin{aligned}
& A_{f}=\left(1-\psi_{y}^{2}\right) \psi_{x x}+2 \psi_{x} \psi_{y} \psi_{x y}+\left(1-\psi_{x}^{2}\right) \psi_{y y}, \\
& B_{f}=1-\psi_{x}^{2}-\psi_{y}^{2} .
\end{aligned}
$$

Since $f$ is a ZMC-graph, the function $A:=A_{f}$ vanishes identically. So we have

$$
0=\left.A_{x x}\right|_{x=0}=2 \alpha \alpha^{\prime}+\alpha^{\prime \prime},
$$

where the prime means the derivative with respect to $y$. Hence there exists a constant $\mu$ such that

$$
\alpha^{\prime}+\alpha^{2}+\mu=0 .
$$

If $\mu>0$, then $f$ is space-like, and if $\mu<0$, then $f$ is time-like. If $\mu=0$, the causal type of $f$ cannot be specified, and the following four possibilities arise:

(i) the graph of $\psi$ is of mixed type,

(ii) the graph of $\psi$ consists of space-like points, except for the $y$-axis,

(iii) the graph of $\psi$ consists of time-like points except for the $y$-axis, and

(iv) the graph of $\psi$ consists only of null points.

Remark 2.1. If we weaken the condition that the image of $f$ contains a null line segment, an example for the case (i) was shown in [7], and a general local existence theorem for such $\psi$ satisfying (i), (ii) and (iii) was shown in [12, Prop. 6.7].

As shown in [6] and [12], by a homothetic change

$$
\begin{aligned}
& \tilde{f}(x, y):=(x, y, \tilde{\psi}(x, y)) \\
& \left(\tilde{\psi}(x, y):=\frac{1}{m} \psi(m x, m y), m>0\right),
\end{aligned}
$$

one can normalize $\mu$ to be $-1,0$ or 1 . In fact, as shown in $[6]$,

$$
\alpha^{+}:=-\tan (y+c) \quad\left(|c|<\frac{\pi}{2}\right)
$$

is a general solution of $\alpha^{\prime}+\alpha^{2}+\mu=0$ for $\mu=1$. If $\mu=0$, then

$$
\alpha_{I}^{0}:=0 \quad \text { and } \quad \alpha_{I I}^{0}:=\frac{1}{y+c} \quad(c \in \boldsymbol{R} \backslash\{0\})
$$

are the solutions, and

$$
\begin{aligned}
\alpha_{I}^{-}:=\tanh (y+c) & (c \in \boldsymbol{R}), \\
\alpha_{I I}^{-}:=\operatorname{coth}(y+c) & (c \in \boldsymbol{R} \backslash\{0\}), \\
\alpha_{I I I}^{-}:= \pm 1 &
\end{aligned}
$$

are the solutions for $\mu=-1$. If 


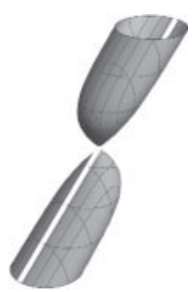

Fig. 3. A time-like ZMC-surface without self-intersection which has a cone-like singular point lying on a null line.

$$
\alpha=\alpha^{+}, \alpha_{I I}^{0}, \alpha_{I I}^{-},
$$

then they are not defined on $\boldsymbol{R}$, so these three cases cannot produce any embedded ZMC-surfaces with entire null lines. In fact, the maximal surface given in (2) is of type $\alpha^{+}$. On the other hand, the light-cone (Fig. 1, right) and the maximal surface given in (1) is of type $\alpha_{I I}^{0}$ on each null line. Moreover, the time-like surface given by the implicit function

$$
(y-t+\tanh t)^{2}+x^{2}=\tanh ^{2} t
$$

has a null line $y=t, x=0$ and is of type $\alpha_{I I}^{-}$(see Fig. 3). So if we seek candidates of ZMC-immersions containing entire null lines, then only the possibilities are

$$
\alpha=\alpha_{I}^{0}, \alpha_{I}^{-}, \alpha_{I I I}^{-} .
$$

The cases $\alpha=\alpha_{I}^{-}, \alpha_{I I I}^{-}$give only time-like ZMCsurfaces, and properly embedded examples were known (cf. [3, Examples 2.2 and 2.3]). So only the case $\alpha:=\alpha_{I}^{0}(\equiv 0)$ is remaining.

This special case was discussed in [7], and here we would like to point out the method used in [7] is sufficient to show the following assertion:

Theorem 2.2. There exist real analytic functions $\psi_{i}: U \rightarrow \boldsymbol{R}(i=1,2,3)$ defined on a domain $U$ in $\boldsymbol{R}^{2}$, each of whose graphs gives a $Z M C$ embedding containing an entire null line of type $\alpha_{I}^{0}$, so that $\psi_{1}, \psi_{2}$ and $\psi_{3}$ satisfy the conditions (i), (ii) and (iii), respectively.

The existence of $\psi_{1}$ and $\psi_{2}$ proves Theorem 1.4 in the introduction.

3. Proof of Theorem 2.2. We first consider the case (i). For each $c>0$, a function $\psi$ of a ZMC surface of mixed type satisfying $\alpha=0$ was constructed in [7]. Such a surface can be characterized by the condition

$$
\beta_{3}(y)=3 c y, \beta_{k}(0)=\beta_{k}^{\prime}(0)=0 \quad(k \geq 4) .
$$

For an arbitrary $\delta>0$, there exists a positive constant $C_{\delta}$ such that $\psi$ is defined on

$$
V_{\delta}:=\left(-C_{\delta}^{-1}, C_{\delta}^{-1}\right) \times(-\delta, \delta) .
$$

Remark 3.1. In [7, Page 290], the inequality

$$
\left|\beta_{l}\right| \leq \frac{3 c|y|^{l^{*}+2}}{\left(l^{*}+2\right)^{2}} M^{l-3} \leq \theta_{0} c^{l}
$$

was shown, where $C_{\delta}:=\delta M$ and $\theta_{0}:=3(\delta M)^{3} / c$. However, there was a typographical error, and we should correct

$$
C_{\delta}:=\sqrt{\delta} M, \quad \theta_{0}:=\frac{3 c}{\sqrt{\delta} M^{3}} .
$$

Then (8) holds, correctly.

The uniqueness of the solution $\psi$ implies that $\psi$ can be extended on the domain $U:=\bigcup_{\delta \geq 1} V_{\delta}$. In particular, $U$ contains the entirety of the $y$-axis, and so $\psi$ gives an example of type (i) containing the entire null line $L$.

We show the existence of surfaces satisfying (ii) or (iii) as a modification of the proof of [7]. We will change the initial condition and modify the estimates in [7] as follows: We set

$$
\begin{aligned}
& \beta_{3}(y)=0, \quad \beta_{4}(y)=4 c y \quad(c \neq 0), \\
& \beta_{k}(0)=\beta_{k}^{\prime}(0)=0 \quad(k \geq 5),
\end{aligned}
$$

as the initial condition. Then, by $[12,(6.5)]$,

$$
B_{f}(x, 0)=-2 c x^{4}+\mathcal{O}\left(x^{5}\right)
$$

holds. Hence, if the power series in (3) with the condition (10) converges, the graph of $\psi$ satisfies (ii) (resp. (iii)) if $c<0$ (resp. $c>0$ ). The convergence of (3) can be proved by imitating the argument in [7]. In [7], functions $b_{i}(i \geq 0)$ were used, where

$$
b_{0}:=y \quad b_{1}:=0, \quad b_{2}:=\alpha(=0)
$$

and $b_{k}=\beta_{k}$ holds for $k \geq 3$. Since $b_{2}=b_{3}=0$ in [7], the series $\left\{P_{k}, Q_{k}, R_{k}\right\}$ were produced by the following recursive formula: We set $P_{j}=Q_{k}=R_{l}=0$ for $3 \leq j \leq 5,3 \leq k \leq 9,3 \leq l \leq 10$, and

$$
\begin{aligned}
& P_{k}:=\sum_{m=4}^{k-2} \frac{2(k-2 m+3)}{k-m+2} \beta_{m} \beta_{k-m+2}^{\prime}, k \geq 6, \\
& Q_{k}:=\sum_{m=4}^{k-6} \sum_{n=4}^{k-m-2} \frac{3 n-k+m-1}{m n} \beta_{m}^{\prime} \beta_{n}^{\prime} \beta_{k-m-n+2}, \\
& k \geq 10,
\end{aligned}
$$




$$
R_{k}:=\sum_{m=4}^{k-7} \sum_{n=4}^{k-m-3} \frac{\beta_{m} \beta_{n} \beta_{k-m-n+2}^{\prime \prime}}{k-m-n+2}, k \geq 11 .
$$

Here $P_{k}, Q_{k}$ and $R_{k}$ are determined by $\beta_{j}(4 \leq j \leq$ $k-2)$. Thus, the functions $\beta_{k}(k \geq 5)$ are inductively determined by the ordinary differential equation

$$
\beta_{k}^{\prime \prime}=-k\left(P_{k}+Q_{k}-R_{k}\right), \beta_{k}(0)=\beta_{k}^{\prime}(0)=0,
$$

where $k \geq 5$. The following proposition holds, which corresponds to [7, Proposition 1.3]:

Proposition 3.2. For each $c \neq 0$ and $\delta>0$, we set

$$
M_{\delta}:=3 \max \left\{144 \tau|c||\delta|^{3 / 2}, \sqrt[4]{192 c^{2} \tau}\right\},
$$

where $\tau$ is a positive constant such that

$$
t \int_{t}^{1-t} \frac{d u}{u^{2}(1-u)^{2}} \leq \tau \quad\left(0<t<\frac{1}{2}\right) .
$$

Then the functions $\beta_{l}(y)(l \geq 5)$ satisfy the inequalities

$$
\begin{aligned}
\left|\beta_{l}^{\prime \prime}(y)\right| & \leq|c||y|^{l^{*}} M^{l-3}, \\
\left|\beta_{l}^{\prime}(y)\right| & \leq \frac{3|c||y|^{l^{*}+1}}{l^{*}+2} M^{l-3}, \\
\left|\beta_{l}(y)\right| & \leq \frac{3|c||y|^{l^{*}+2}}{\left(l^{*}+2\right)^{2}} M^{l-3}
\end{aligned}
$$

for any $y \in[-\delta, \delta]$, where

$$
l^{*}:=\frac{1}{2}(l-1)-2 \quad(l=5,6, \ldots) .
$$

Proof. We can prove this by induction on $l \geq 5$. The functions $\beta_{k}$ of small indices are determined by the recursive formula (11) as follows:

$$
\begin{aligned}
& \beta_{4}=4 c y, \quad \beta_{5}=0, \quad \beta_{6}=-8 c^{2} y^{3}, \\
& \beta_{7}=0, \quad \beta_{8}=-32 c^{3} y^{5} .
\end{aligned}
$$

The estimates (13)-(15) for $l=5$ follow from (16). In the case of [7], we set $b_{3}\left(=\beta_{3}\right) \neq 0$, but in our present case $b_{3}=0$ and the first non-trivial term begins from $b_{4}\left(=\beta_{4}\right)$. In particular, sub-terms appearing in $P_{k}, Q_{k}, R_{k}$ are fewer than those in [7]. So, (13)-(15) for $l \geq 6$ follow using the same induction argument as in [7]. Therefore, the same estimates as in the proof of Proposition 1.9 in [7] are entirely valid also in this case.

By Proposition 3.2, for an arbitrary $\delta \geq 1, \psi$ is well-defined on

$$
V_{\delta}:=\left(-C_{\delta}^{-1}, C_{\delta}^{-1}\right) \times(-\delta, \delta),
$$

where $C_{\delta}:=\sqrt{\delta} M$ (cf. (9)). In fact, like as in the case of [7], the inequality

$$
\left|\beta_{l}\right| \leq \frac{3|c||y|^{l^{*}+2}}{\left(l^{*}+2\right)^{2}} M^{l-3} \leq \theta_{0} C_{\delta}^{l} \quad(l \geq 5)
$$

can be shown, where $\theta_{0}:=3|c| /\left(\sqrt{\delta} M^{3}\right)$. The uniqueness of the solution $\psi$ implies that $\psi$ can be extended on the domain

$$
U:=\bigcup_{\delta \geq 1} V_{\delta}
$$

In particular, $U$ contains the entirety of the $y$-axis, and so $\psi$ gives an example of type (ii) (resp. (iii)) if $c<0$ (resp. $c>0$ ) containing the entire null line $L$.

Remark 3.3. The domain $U$ defined in (18) is not convex in $\boldsymbol{R}^{2}$. However, this fact is crucial to construct a maximal graph containing an entire line. In fact, as pointed out in [4, Lemma 1], if there exists a maximal graph defined on a closed convex domain in $\boldsymbol{R}^{2}$ whose image contains an entire null line, then it must be a light-like plane. So if there exists an entire graph of mixed type containing an entire null line $L$, then either

- the both side of the entire null line are timelike, or

- there exists a sequence of time-like points (on the side containing space-like points) which is asymptotic to the line $L$,

as a consequence.

Acknowledgement. The authors would like to express their gratitude to Dr. Atsufumi Honda and Dr. Wayne Rossman for helpful comments.

\section{References}

[ 1 ] S. Akamine, Causal characters of zero mean curvature surfaces of Riemann type in the Lorentz-Minkowski 3-space, Kyushu J. Math. 71 (2017), no. 2, 211-249.

[ 2 ] S. Akamine and R. K. Singh, Wick rotations of solutions to the minimal surface equation, the zero mean curvature equation and the BornInfeld equation, Proc. Indian Acad. Sci. Math. Sci. 129 (2019), no. 3, Art. 35.

[ 3 ] S. Akamine, M. Umehara and K. Yamada, Improvement of the Bernstein-type theorem for space-like zero mean curvature graphs in Lorentz-Minkowski space using fluid mechanical duality, (to appear in Proc. Amer. Math. Soc.).

[ 4 ] S. Akamine, A. Honda, M. Umehara and K. Yamada, Bernstein-type theorem for zero mean curvature hypersurfaces without timelike points in Lorentz-Minkowski space, arXiv:1907.01754. 
[ 5 ] S. Y. Cheng and S. T. Yau, Maximal space-like hypersurfaces in the Lorentz-Minkowski spaces, Ann. of Math. (2) 104 (1976), no. 3, 407-419.

6 ] S. Fujimori, Y. W. Kim, S.-E. Koh, W. Rossman, H. Shin, H. Takahashi, M. Umehara, K. Yamada and S.-D. Yang, Zero mean curvature surfaces in $\mathbf{L}^{3}$ containing a light-like line, C. R. Math. Acad. Sci. Paris 350 (2012), no. 21-22, 975-978.

[ 7 ] S. Fujimori, Y. W. Kim, S.-E. Koh, W. Rossman, H. Shin, M. Umehara, K. Yamada and S.-D. Yang, Zero mean curvature surfaces in LorentzMinkowski 3-space which change type across a light-like line, Osaka J. Math. 52 (2015), no. 1, 285-297, Erratum: Osaka J. Math. 53 (2016), no. $1,289-292$.

[ 8 ] K. Hashimoto and S. Kato, Bicomplex extensions of zero mean curvature surfaces in $\mathbf{R}^{2,1}$ and $\mathbf{R}^{2,2}$, J. Geom. Phys. 138 (2019), 223-240.
[ 9 ] V. A. Klyachin, Zero mean curvature surfaces of mixed type in Minkowski space, Izv. Math. 67 (2003), no. 2, 209-224; translated from Izv. Ross. Akad. Nauk Ser. Mat. 67 (2003), no. 2, $5-20$.

[ 10 ] O. Kobayashi, Maximal surfaces in the 3-dimensional Minkowski space $L^{3}$, Tokyo J. Math. 6 (1983), no. 2, 297-309.

[11 M. Umehara and K. Yamada, Maximal surfaces with singularities in Minkowski space, Hokkaido Math. J. 35 (2006), no. 1, 13-40.

[ 12 ] M. Umehara and K. Yamada, Surfaces with lightlike points in Lorentz-Minkowski 3-space with applications, in Lorentzian geometry and related topics, Springer Proc. Math. Stat., 211, Springer, Cham, 2017, pp. 253-273.

[13] M. Umehara and K. Yamada, Hypersurfaces with light-like points in a Lorentzian manifold, J. Geom. Anal. 29 (2019), 3405-3437. 